\title{
First Assessment of the Binary Lens OGLE-2015-BLG-0232
}

Bachelet, E.; Bozza, V.; Han, C.; Udalski, A.; Bond, I. A.; Beaulieu, J. -P.; Street, R. A.; Kim, H. -l; Bramich, D. M.; Cassan, A.; Dominik, M.; Jaimes, R. Figuera; Horne, K.; Hundertmark, M.; Mao, S.; Menzies, J.; Ranc, C.; Schmidt, R.; Snodgrass, C.; Steele, I. A.; Tsapras, Y.; Wambsganss, J.; Mroz, P.; Soszynski, I.; Szymanski, M. K.; Skowron, J.; Pietrukowicz, P.; Kozlowski, S.; Poleski, R.; Ulaczyk, K.; Pawlak, M.; Abe, F.; Barry, R.; Bennett, D. P.; Bhattacharya, A.; Donachie, M.; Fukui, A.; Hirao, Y.; Itow, Y.; Kawasaki, K.; Kondo, I.; Koshimoto, N.; Li, M. Cheung Alex; Matsubara, Y.; Muraki, Y.; Miyazaki, S.; Nagakane, M.; Rattenbury, N. J.; Suematsu, H.; Sullivan, D. J.; OGLE Collaboration; FUN Collaboration; MOA Collaboration; PLANET RoboNet Collaboration

Published in:

Astrophysical Journal Supplement Series

DOI:

10.3847/1538-4357/aaedb9

Publication date:

2019

Document version

Publisher's PDF, also known as Version of record

Document license:

CC BY-ND

Citation for published version (APA):

Bachelet, E., Bozza, V., Han, C., Udalski, A., Bond, I. A., Beaulieu, J. -P., Street, R. A., Kim, H. -I., Bramich, D. M., Cassan, A., Dominik, M., Jaimes, R. F., Horne, K., Hundertmark, M., Mao, S., Menzies, J., Ranc, C., Schmidt, R., Snodgrass, C., ... OGLE Collaboration; FUN Collaboration; MOA Collaboration; PLANET RoboNet Collaboration (2019). First Assessment of the Binary Lens OGLE-2015-BLG-0232. Astrophysical Journal Supplement Series, 870(1), [11]. https://doi.org/10.3847/1538-4357/aaedb9 


\section{Open Research Online}

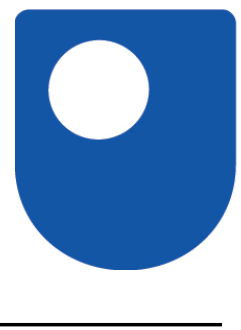

The Open University's repository of research publications and other research outputs

\section{First Assessment of the Binary Lens OGLE-2015-BLG-0232}

\section{Journal Item}

\section{How to cite:}

Bachelet, E.; Bozza, V.; Han, C.; Udalski, A.; Bond, I. A.; Beaulieu, J.-P.; Street, R. A.; Kim, H.-I; Bramich, D. M.; Cassan, A.; Dominik, M.; Jaimes, R. Figuera; Horne, K.; Hundertmark, M.; Mao, S.; Menzies, J.; Ranc, C.; Schmidt, R.; Snodgrass, C.; Steele, I. A.; Tsapras, Y.; Wambsganss, J.; Mróz, P.; Soszyński, I.; Szymański, M. K.; Skowron, J.; Pietrukowicz, P.; Kozłowski, S.; Poleski, R.; Ulaczyk, K.; Pawlak, M.; Abe, F.; Barry, R.; Bennett, D. P.; Bhattacharya, A.; Donachie, M.; Fukui, A.; Hirao, Y.; Itow, Y.; Kawasaki, K.; Kondo, I.; Koshimoto, N.; Cheung Alex Li, M.; Matsubara, Y.; Muraki, Y.; Miyazaki, S.; Nagakane, M.; Rattenbury, N. J.; Suematsu, H.; Sullivan, D. J.; Sumi, T.; Suzuki, D.; Tristram, P. J. and Yonehara, A. (2018). First Assessment of the Binary Lens OGLE-2015-BLG-0232. The Astrophysical Journal, 870(1), article no. 11.

For guidance on citations see FAQs

(C) [not recorded]

Version: Version of Record

Link(s) to article on publisher's website:

http://dx.doi.org/doi:10.3847/1538-4357/aaedb9

Copyright and Moral Rights for the articles on this site are retained by the individual authors and/or other copyright owners. For more information on Open Research Online's data policy on reuse of materials please consult the policies page.

\section{oro.open.ac.uk}




\title{
First Assessment of the Binary Lens OGLE-2015-BLG-0232
}

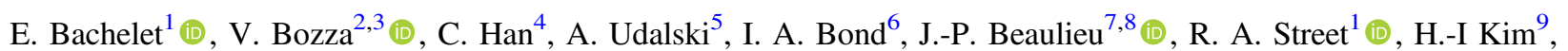

D. M. Bramich ${ }^{10}$, A. Cassan ${ }^{11}$, M. Dominik ${ }^{12,36}$, R. Figuera Jaimes ${ }^{12,13,14}$, K. Horne ${ }^{12}$ (1), M. Hundertmark ${ }^{15,16}$ (1) , S. Mao ${ }^{13,17,18,19}$,

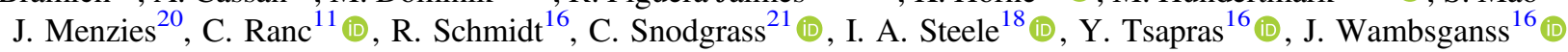
(The RoboNet collaboration),

P. Mróz ${ }^{5}$ (10) I. Soszyński ${ }^{5}$, M. K. Szymański ${ }^{5}$, J. Skowron ${ }^{5}$ (1), P. Pietrukowicz ${ }^{5}$ (1) S. Kozłowski ${ }^{5}$, R. Poleski ${ }^{22}$, K. Ulaczyk $^{5}$ (D), M. Pawlak ${ }^{5}$

(The OGLE collaboration),

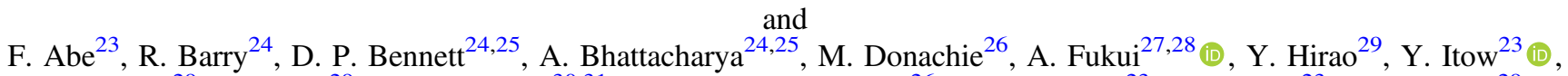
K. Kawasaki ${ }^{29}$, I. Kondo ${ }^{29}$, N. Koshimoto ${ }^{30,31}$ (1) M. Cheung Alex Li ${ }^{26}$, Y. Matsubara ${ }^{23}$, Y. Muraki ${ }^{23}$, S. Miyazaki ${ }^{29}$ (i), M. Nagakane ${ }^{29}$, N. J. Rattenbury ${ }^{26}$ (D) H. Suematsu ${ }^{29}$, D. J. Sullivan ${ }^{32}$, T. Sumi ${ }^{29}$, D. Suzuki ${ }^{33}$ (1) P. J. Tristram ${ }^{34}$, and A. Yonehara ${ }^{35}$

(The MOA collaboration)

\footnotetext{
${ }^{1}$ Las Cumbres Observatory, 6740 Cortona Drive, Suite 102, Goleta, CA 93117 USA

${ }^{2}$ Dipartimento di Fisica "E.R. Caianiello", Università di Salerno, Via Giovanni Paolo II 132, I-84084, Fisciano, Italy

${ }^{3}$ Istituto Internazionale per gli Alti Studi Scientifici (IIASS), Via G. Pellegrino 19, I-84019 Vietri sul Mare (SA), Italy

${ }_{5}^{4}$ Department of Physics, Chungbuk National University, Cheongju 28644, Korea

${ }^{5}$ Warsaw University Observatory, Al. Ujazdowskie 4, 00-478 Warszawa, Poland

${ }^{6}$ Institute of Natural and Mathematical Sciences, Massey University, Auckland 0745, New Zealand

${ }^{7}$ School of Physical Sciences, University of Tasmania, Private Bag 37 Hobart, Tasmania 7001 Australia; jeanphilippe.beaulieu@utas.edu.au

${ }^{8}$ Sorbonne Universités, UPMC Université Paris 6 et CNRS, UMR 7095, Institut d'Astrophysique de Paris, 98 bis bd Arago, F-75014 Paris, France; beaulieu @iap.fr ${ }^{9}$ Korea Astronomy and Space Science Institute, Daejon 34055, Korea

${ }^{10}$ New York University Abu Dhabi, PO Box 129188, Saadiyat Island, Abu Dhabi, UAE

${ }^{11}$ Sorbonne Universités, UPMC Univ Paris 6 et CNRS, UMR 7095, Institut d'Astrophysique de Paris, 98 bis bd Arago, F-75014 Paris, France

${ }^{12}$ SUPA, School of Physics \& Astronomy, University of St Andrews, North Haugh, St Andrews KY16 9SS, UK

${ }^{13}$ Physics Department and Tsinghua Centre for Astrophysics, Tsinghua University, Beijing 100084, People's Republic of China

${ }^{14}$ European Southern Observatory, Karl-Schwarzschild-Str. 2, D-85748 Garching bei München, Germany

${ }^{15}$ Niels Bohr Institute \& Centre for Star and Planet Formation, University of Copenhagen, Øster Voldgade 5, DK-1350-Copenhagen K, Denmark

${ }^{16}$ Zentrum für Astronomie der Universität Heidelberg, Astronomisches Rechen-Institut, Mönchhofstr. 12-14, D-69120 Heidelberg, Germany

${ }^{17}$ National Astronomical Observatories, Chinese Academy of Sciences, 20A Datun Road, Chaoyang District, Beijing 100012, People's Republic of China

18 Jodrell Bank Centre for Astrophysics, School of Physics and Astronomy, The University of Manchester, Oxford Road, Manchester M13 9PL, UK ${ }^{19}$ South African Astronomical Observatory, PO Box 9, Observatory 7935, South Africa

${ }^{20}$ Planetary and Space Sciences, Department of Physical Sciences, The Open University, Milton Keynes, MK7 6AA, UK

${ }^{21}$ Astrophysics Research Institute, Liverpool John Moores University, Liverpool CH41 1LD, UK

${ }^{22}$ Department of Astronomy, Ohio State University, 140 W. 18th Avenue,Columbus, OH 43210, USA

${ }^{23}$ Institute for Space-Earth Environmental Research, Nagoya University, Nagoya 464-8601, Japan ${ }^{24}$ Code 667, NASA Goddard Space Flight Center, Greenbelt, MD 20771, USA

${ }^{25}$ Department of Astronomy, University of Maryland, College Park, MD 20742, USA

${ }^{26}$ Department of Physics, University of Auckland, Private Bag 92019, Auckland, New Zealand

${ }^{27}$ Subaru Telescope Okayama Branch Office, National Astronomical Observatory of Japan, NINS, 3037-5 Honjo, Kamogata, Asakuchi, Okayama 719-0232, Japan

${ }^{28}$ Instituto de Astrofísica de Canarias, Vía Láctea s/n, E-38205 La Laguna, Tenerife, Spain

${ }^{29}$ Department of Earth and Space Science, Graduate School of Science, Osaka University, Toyonaka, Osaka 560-0043, Japan

${ }^{30}$ Department of Astronomy, Graduate School of Science, The University of Tokyo, 7-3-1 Hongo, Bunkyo-ku, Tokyo 113-0033, Japan

${ }^{31}$ National Astronomical Observatory of Japan, 2-21-1 Osawa, Mitaka, Tokyo 181-8588, Japan

${ }^{32}$ School of Chemical and Physical Sciences, Victoria University, Wellington, New Zealand

${ }^{33}$ Institute of Space and Astronautical Science, Japan Aerospace Exploration Agency, 3-1-1 Yoshinodai, Chuo, Sagamihara, Kanagawa, 252-5210, Japan

${ }_{35}^{34}$ University of Canterbury Mt. John Observatory, P.O. Box 56, Lake Tekapo 8770, New Zealand

${ }^{35}$ Department of Physics, Faculty of Science, Kyoto Sangyo University, 603-8555 Kyoto, Japan

Received 2018 July 19; revised 2018 October 5; accepted 2018 October 25; published 2018 December 27
}

\begin{abstract}
We present an analysis of the microlensing event OGLE-2015-BLG-0232. This event is challenging to characterize for two reasons. First, the light curve is not well sampled during the caustic crossing due to the proximity of the full Moon impacting the photometry quality. Moreover, the source brightness is difficult to estimate because this event is blended with a nearby $\mathrm{K}$ dwarf star. We found that the light-curve deviations are likely due to a close brown dwarf companion (i.e., $s=0.55$ and $q=0.06$ ), but the exact nature of the lens is still unknown. We finally discuss the potential of follow-up observations to estimate the lens mass and distance in the future.
\end{abstract}

Key words: binaries: general - gravitational lensing: micro

\footnotetext{
${ }^{36}$ Royal Society University Research Fellow.
} 


\section{Introduction}

Twenty years after the first exoplanet detection, it is clear that planets are abundant in the Milky Way (Cassan et al. 2012; Bonfils et al. 2013; Fressin et al. 2013; Clanton \& Gaudi 2016; Suzuki et al. 2016). But the dividing line between superJupiters and brown dwarfs is still uncertain. Burrows et al. (2001) define brown dwarfs as objects within mass limits $[13,73] M_{J}$. As underlined by Schlaufman (2018), this definition is problematic because the critical mass for deuterium burning depends on the object composition (Spiegel et al. 2011). More recently, an alternative definition has been proposed based on the formation mechanisms (Schneider et al. 2011): planets are formed by core accretion, while brown dwarfs are a result of gas-collapse. The former is motivated by exoplanet formation models and by the observational evidence that giant planets tend to form more frequently around metalrich stars (Buchhave et al. 2012; Mordasini et al. 2012; Mortier et al. 2012). In contrast, Latham et al. (2002) found no significant correlation between metallicity and stellar binary occurrence. But this definition is also problematic because it is nearly impossible to distinguish the two scenarios observationally (Wright et al. 2011; Bryan et al. 2018). Recently, Schlaufman (2018) revisited the mass definition by combining and clustering samples of low-mass stars, brown dwarfs, and planets orbiting solar-type stars and ultimately derived a surprisingly low upper planetary mass limit of $\sim 6 M_{J}$.

Brown dwarf detections are therefore important to understand the planetary regime boundaries but these objects are intrinsically difficult to detect directly, due to their lowluminosity. Moreover, the radii of brown dwarfs and Jupiterlike planets are very similar due to the degeneracy pressure (Zapolsky \& Salpeter 1969; Burrows \& Liebert 1993). It is therefore difficult to distinguish them with the transit method alone. Microlensing, however, can detect brown dwarfs several kiloparsecs away, either in binary systems or as single objects (Zhu et al. 2016; Chung et al. 2017; Shvartzvald et al. 2018), because the method does not need flux measurements from the lens. Several brown dwarfs and brown dwarf candidates have been discovered through this method (Bachelet et al. 2012a; Bozza et al. 2012; Ranc et al. 2015; Han et al. 2016; Mróz et al. 2017; Poleski et al. 2017).

In this work, we present the analysis of OGLE-2015-BLG0232/MOA-2015-BLG-046. The data presented in Section 2 show clear signatures of a binary lens event. In Section 3, we present the modeling procedure and find that the mass ratio of the lens system favors a brown dwarf companion (close model) or a low-mass $\mathrm{M}$ dwarf companion (wide model). We present a detailed study of both the microlensing source and the bright blend in Section 4. Because no parallax was measured, we discuss in Section 6 the possible follow-up observations to unlock the final solution of this microlensing puzzle.

\section{Observations}

The microlensing event OGLE-2015-BLG-0232 $(\alpha=$ $18^{\mathrm{h}} 06^{\mathrm{m}} 43^{\mathrm{s}} .84, \quad \delta=-32^{\circ} 54^{\mathrm{m}} 27^{\mathrm{s}} .3 ; \quad l=-1^{\circ} .172199, \quad \mathrm{~b}=$ $\left.-5^{\circ} .9060\right)$ was an early event of the 2015 microlensing season first discovered by the Optical Gravitational Lens Experiment (OGLE) (Udalski 2003) on 2015 March 2 UT 17:50 and also detected later by the Microlensing Observations in Astrophysics (MOA) collaboration (Bond et al. 2001) as MOA2015-BLG-046 on 2015 March 10 at UT 16:42. C. Han first delivered an email alert indicating an ongoing anomaly on 2015 March 15 at UT 02:16. Independently, the RoboNet team, based on the SIGNALMEN anomaly detector (Dominik et al. 2007) and the RoboTAP algorithm (Hundertmark et al. 2018), automatically triggered observations on the Las Cumbres Observatory network of robotic telescopes (Tsapras et al. 2009). Unfortunately, the Moon was nearly full during this period, preventing surveys from acquiring more data during the anomaly. This event was also observed in the near-infrared by the VISTA Variables in the Via Lactea (VVV) survey (Minniti et al. 2010). Real-time modeling conducted independently by C.Han and V.Bozza indicated that this event was probably due to a low-mass binary lens $(q \sim 0.01)$. All teams reprocessed their photometry at the end of the season using the difference image analysis (DIA) technique : RoboNet used DanDIA (Bramich 2008; Bramich et al. 2013), and OGLE and MOA used their own implementation of DIA (Udalski et al. 2015; Bond et al. 2001). The $K$ band of VVV was re-reduced using pySIS (Albrow et al. 2009). The VVV pySIS photometry were roughly calibrated to an independent VVV catalog (Beaulieu et al. 2016) by adding an offset of $0.6 \mathrm{mag}$. Note that the $\mathrm{VVV}_{\mathrm{K}}$ light curve is nearly flat, so we did not use this data set in the first round of modeling. In total, 7659 data points are available for the analysis, as summarized in Table 1.

\section{Modeling \\ 3.1. Description}

This event is clearly anomalous and real-time models found that a binary lens with a small mass ratio accurately reproduces the observations. A static binary model is described with seven parameters: $t_{0}$, the time of the minimum impact parameter, $u_{0}$, $t_{\mathrm{E}}=\theta_{\mathrm{E}} / \mu$, the angular Einstein radius crossing time, $\rho=\theta_{*} / \theta_{\mathrm{E}}$, the normalized angular source radius, $s$, the normalized projected separation, $q$, the mass ratio between the two lens components, and finally $\alpha$, the lens/source trajectory angle relative to the binary axis. Here, $\mu$ is the relative proper motion between the source and the lens and $\theta_{\mathrm{E}}$ is the angular Einstein ring; see, for example, Gould (2000). Note that we restrict the modeling of the data points to the time window $t \in[2456850,2457200]$ to speed up the modeling. For events like OGLE-2015-BLG-0232 that exhibit caustic crossings, the limb-darkening of the source star has to be considered. Unfortunately, in this case, the observations taken around HJD $\sim 2457087$ were in the SDSS-i' band only in order to reduce the impact of the moonlight. Moreover, the caustic crossings are not intensively covered by the data. For these reasons, we investigated a simpler model, the Uniform Source Binary Lens (Bozza 2010; Bozza et al. 2012) and use pyLIMA (Bachelet et al. 2017) to perform the modeling. A detailed description of this binary fitting code is given in E. Bachelet (2018, in preparation). We did not use the standard grid approach to locate the global minimum, but instead ran a global search on all parameters using the differential evolution method (Storn \& Price 1997; Bachelet et al. 2017). Briefly, this method uses a set of starting points in parameter space and maintains an ordered population of candidate solutions while exploring potential new solutions by combining existing ones. This algorithm was successfully tested by applying it to previously published events. In practice, we split the parameter space in two regions: $s<1$ and $s>1$. This is motivated by the dramatic change of the caustics topology between these two 
Table 1

Summary of Observations

\begin{tabular}{|c|c|c|c|c|c|c|c|c|}
\hline Name & Collaboration & Location & Aperture(m) & Filter & Code & $N_{\text {data }}$ & Longitude(deg) & Latitude(deg) \\
\hline OGLE $_{I}$ & OGLE & Chile & 1.3 & I & Woźniak & 525 & 289.307 & -29.015 \\
\hline $\mathrm{MOA}_{\mathrm{Red}}$ & MOA & New Zealand & 1.8 & Red & Bond & 6569 & 170.465 & 43.987 \\
\hline $\mathrm{MOA}_{\mathrm{V}}$ & Boller\&Chivens & New Zealand & 0.6 & $\mathrm{~V}$ & Bond & 184 & 170.465 & 43.987 \\
\hline $\operatorname{VVV}_{\mathrm{K}}$ & VISTA & Chile & 4.1 & $\mathrm{~K}$ & pySIS & 198 & 289.6081 & -24.616 \\
\hline $\mathrm{LSCA}_{\mathrm{i}}$ & RoboNet & Chile & 1.0 & SDSS-i & DanDIA & 30 & 289.195 & -30.167 \\
\hline $\mathrm{LSCB}_{\mathrm{i}}$ & RoboNet & Chile & 1.0 & SDSS-i & DanDIA & 23 & 289.195 & -30.167 \\
\hline $\mathrm{LSCC}_{\mathrm{i}}$ & RoboNet & Chile & 1.0 & SDSS-i & DanDIA & 21 & 289.195 & -30.167 \\
\hline $\mathrm{CPTA}_{\mathrm{i}}$ & RoboNet & South Africa & 1.0 & SDSS-i & DanDIA & 21 & 220.810 & -32.347 \\
\hline $\mathrm{CPTB}_{\mathrm{i}}$ & RoboNet & South Africa & 1.0 & SDSS-i & DanDIA & 21 & 220.810 & -32.347 \\
\hline $\mathrm{CPTC}_{\mathrm{i}}$ & RoboNet & South Africa & 1.0 & SDSS-i & DanDIA & 12 & 220.810 & -32.347 \\
\hline $\mathrm{COJA}_{\mathrm{i}}$ & RoboNet & Australia & 1.0 & SDSS-i & DanDIA & 29 & 149.065 & -31.273 \\
\hline $\mathrm{COJB}_{\mathrm{i}}$ & RoboNet & Australia & 1.0 & SDSS-i & DanDIA & 18 & 149.065 & -31.273 \\
\hline
\end{tabular}

regimes and also the presence of the close/wide degeneracy; see, for example, Erdl \& Schneider (1993), Dominik (1999), Bozza (2000), and Cassan (2008). We ran the algorithm several times and found that it converged on similar solutions. This event was also modeled in real time by V.Bozza using RTModel. ${ }^{37}$ This system uses a different method to explore the parameter space: a template matching approach (Mao \& Di Stefano 1995; Liebig et al. 2015). It also found similar solutions, raising our confidence in our results. Results relative to this first exploration can be seen in Table 3.

\subsection{Error Bar Rescaling}

It is common practice to rescale the uncertainties in microlensing using (in mag units in the present work):

$$
\sigma^{\prime}=k \sqrt{\sigma^{2}+e_{\min }^{2}},
$$

where $\sigma^{\prime}$ is the rescaled uncertainty, and $k$ and $e_{\min }$ are parameters that need to be tuned to reach a certain metric to optimize. The usual metric used is to force the $\chi^{2} /$ dof for each data set to converge to 1 (Bachelet et al. 2012b; Miyake et al. 2012; Yee et al. 2013). However, Andrae et al. (2010) showed that the use of the reduced $\chi^{2}$, for model diagnostics, is relevant only for linear models, which is not the case in the present work. Instead, they recommend the use of normality tests of residuals, as seen in Bachelet et al. (2015).

The physical reasons that motivate the rescaling are to account for photometric low-level systematics and potential underestimation of the uncertainties. There are multiple causes coming from both instrumentation and software reductions. The impact is expected to be different for each data set, therefore instead of automatically rescaling the error bars of each data set blindly, we assessed whether this was necessary. To do so, we use the approach described below.

First, we rescaled OGLE-IV uncertainties using the custom method of Skowron et al. (2016). ${ }^{38} \mathrm{We}$ then analyzed the residuals around the best model using three tests of normality: a Kolmogorov-Smirnov test, an Anderson-Darling test, and a Shapiro-Wilk test. We considered rescaling a data set if any of these tests were not successful (i.e., the p-value associated with the test was less than 1\%). All data sets except for MOA $\mathrm{Aed}_{\mathrm{R}}$ passed the three normality tests. The majority of the data sets present a relatively small number of observations $(\leqslant 100)$; any

\footnotetext{
37 http://www.fisica.unisa.it/GravitationAstrophysics/RTModel.htm.

38 http://ogle.astrouw.edu.pl/ogle4/errorbars/blg/errcorr-OIV-BLG-I.dat
}

Table 2

Error Bar Rescaling Coefficients Used in This Paper

\begin{tabular}{lccc}
\hline \hline Name & $N_{\text {data }}$ & $\mathrm{k}$ & $e_{\text {min }}$ \\
\hline OGLE $_{\mathrm{I}}^{a}$ & 68 & $0.97 \pm 0.06$ & 0.0 \\
MOA $_{\text {Red }}$ & 467 & $3.51 \pm 0.08$ & 0.0 \\
MOA $_{\mathrm{V}}$ & 43 & $2.1 \pm 0.2$ & 0.0 \\
VVV $_{\mathrm{K}}$ & 14 & $3.5 \pm 0.5$ & 0.0 \\
$\mathrm{LSCA}_{i}$ & 30 & $1.1 \pm 0.1$ & 0.0 \\
$\mathrm{LSCB}_{\mathrm{i}}$ & 23 & $1.9 \pm 0.2$ & 0.0 \\
$\mathrm{LSCC}_{i}$ & 21 & $1.7 \pm 0.2$ & 0.0 \\
$\mathrm{CPTA}_{i}$ & 21 & $1.1 \pm 0.1$ & 0.0 \\
$\mathrm{CPTB}_{i}$ & 21 & $1.2 \pm 0.1$ & 0.0 \\
$\mathrm{CPTC}_{i}$ & 12 & $1.2 \pm 0.2$ & 0.0 \\
$\mathrm{COJA}_{i}$ & 29 & $1.7 \pm 0.1$ & 0.0 \\
$\mathrm{COJB}_{i}$ & 18 & $2.1 \pm 0.3$ & 0.0 \\
\hline
\end{tabular}

Note. ${ }^{a}$ Note that the OGLE $_{\mathrm{I}}$ uncertainties receive special treatment before this rescaling step; see the text.

deviations to normality would thus be hard to detect. On the other hand, that might indicate that uncertainties reproduce the data scatter accurately. Note that the OGLE-IV data set also passed the three tests after the rescaling process.

As a secondary check, we follow the same approach as Dominik et al. (2018) and fit the parameters of Equation (1) around the best model from the previous section, using the modified $\chi^{2^{\prime}}$ :

$$
\chi^{2^{\prime}}=\sum_{i} \frac{\left(f_{i}-m_{i}\right)^{2}}{\sigma_{i}^{\prime 2}}+2 \ln \left(\sigma_{i}^{\prime}\right),
$$

with being $f_{i}$ the observed flux, $m_{i}$ being the microlensing model in flux, and $\sigma_{i}^{\prime}$ being the modified error in flux relative to Equation (1). It was immediately clear that the term $e_{\min }$ was not constrained, due to the relatively small range of magnification in the light curves. We therefore delete this term from Equation (1) and fit only the first term $k$. The results presented in the Table 2 are consistent with the previous analysis and indicate a soft rescaling, with the exception of the $\mathrm{MOA}_{\text {Red }}$ data set.

\subsection{Results}

Both algorithms converged to models with similar geometries: the strong anomalies seen in Figure 1 are due to a central caustic crossing. However, the data do not constrain strongly 


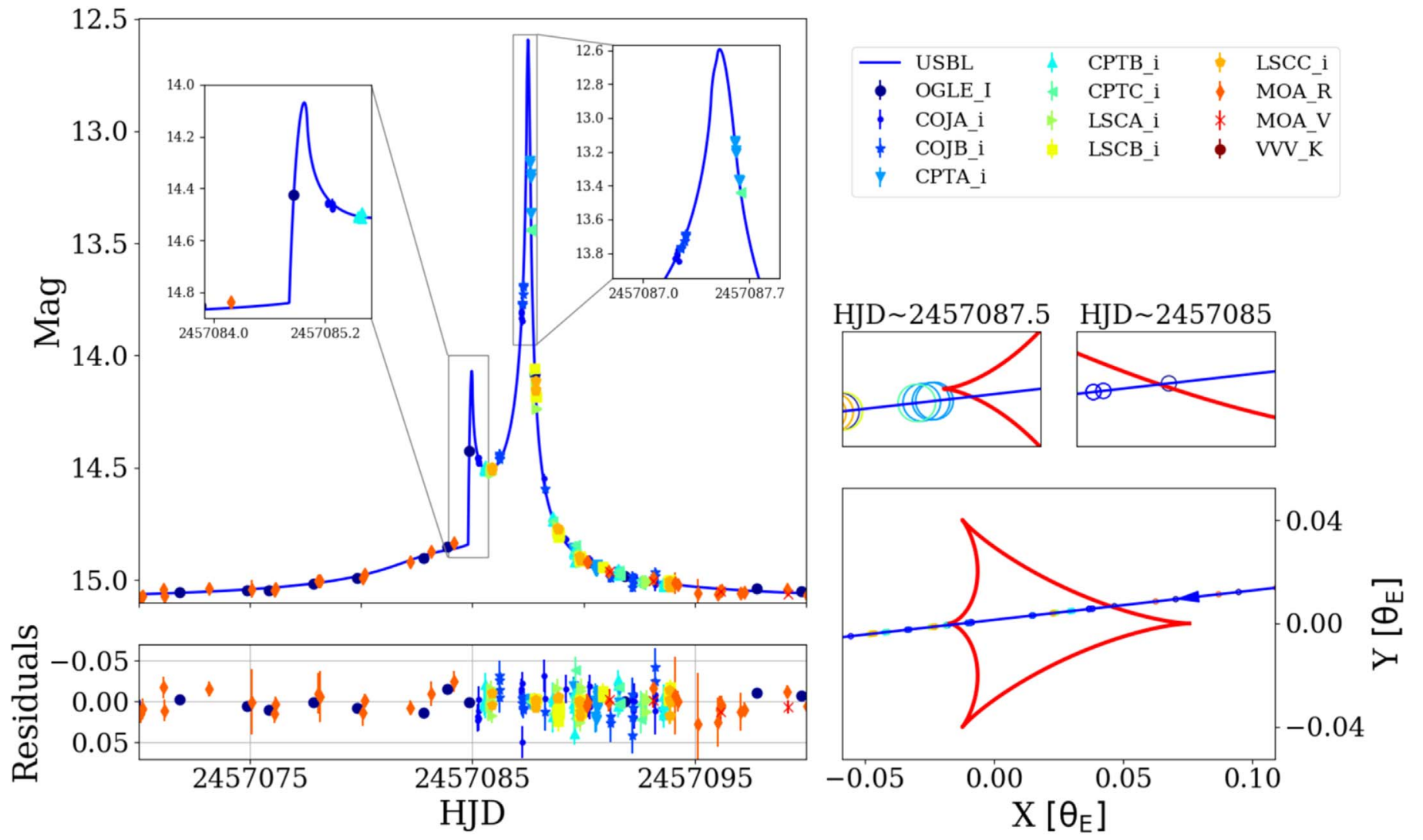

Figure 1. Left: light curves and best-fit model for OGLE-2015-BLG-0232. Right: central caustic (red curve), source trajectory (blue line), and source positions at the epoch of observations. The insets show zoomed-in views around the caustic crossings. There was one OGLE $_{I}$ measurement during the caustic entry and four CPTA $_{i}$ points (only two are visible in the inset) during the cusp exit. This allows a reasonable constraint on the normalized source radius $\rho$.

Table 3

Close/Wide Best Models of pyLIMA, RTModel, and MCMC Explorations

\begin{tabular}{|c|c|c|c|c|c|c|}
\hline Parameters & pyLIMA $(s<1)$ & $\operatorname{RTModel}^{\mathrm{a}}(s<1)$ & $\operatorname{MCMC}(s<1)$ & pyLIMA $(s>1)$ & $\operatorname{RTModel}^{\mathrm{a}}(s>1)$ & $\overline{\operatorname{MCMC}(s>1)}$ \\
\hline$t_{c}-2450000$ & $7087.20(1)$ & $7087.49(4)$ & $7087.2(2)$ & $7086.68(4)$ & $7086.93(4)$ & $7086.76(8)$ \\
\hline$u_{c}$ & $-0.00048(4)$ & $0.00135(7)$ & $-0.0005(6)$ & $0.00320(8)$ & $0.0020(2)$ & $0.0026(5)$ \\
\hline$t_{E}$ & $41.7(3)$ & $46.1(3)$ & $42(6)$ & $34.7(1)$ & $35(3)$ & $39(3)$ \\
\hline$\rho\left(10^{-4}\right)$ & $9.9(3)$ & $19.9(8)$ & $10(1)$ & $7.0(5)$ & $7.0(2)$ & $7.3(9)$ \\
\hline$s$ & $0.545(2)$ & $0.699(2)$ & $0.55(7)$ & $3.05(1)$ & $2.58(2)$ & $2.9(2)$ \\
\hline$q$ & $0.0597(8)$ & $0.0180(1)$ & $0.06(2)$ & $0.338(3)$ & $0.17(1)$ & $0.24(6)$ \\
\hline$\alpha$ & $-3.031(3)$ & $-3.061(2)$ & $-3.03(2)$ & $3.017(4)$ & $3.045(5)$ & $3.008(9)$ \\
\hline$\chi^{2}$ & 766 & 799 & 764 & 822 & 843 & 812 \\
\hline
\end{tabular}

Note. Models from the RTModel were used as a starting point for a Levenberg-Marquardt (LM) optimization with pyLIMA. Numbers in brackets in the table represent $1 \sigma$ errors from $\mathrm{LM}$ and the $68 \%$ range for the MCMC explorations.

a The parameters are obtained from the online RTModel website (http://www.fisica.unisa.it/gravitationAstrophysics/RTModel/2015/RTModel.htm).

the models, leading to significant differences in the model parameters given in the Table 3. To obtain a more comprehensive picture, we run two sets of Monte-Carlo Markov Chain explorations around these best models, using the emcee algorithm (Foreman-Mackey et al. 2013) implemented in pyLIMA. Note that during this optimization process, we modified the model parameters so that we model $t_{c}$ and $u_{c}$, the time and closest approach to the central caustic, respectively, instead of $t_{0}$ and $u_{0}$. The idea is to use parameters more directly related to the main features of the light curve. This is a standard practice that significantly improves the model convergence (Cassan 2008; Han 2009; Penny 2014).
The geometry of the best-fitting model is sensitive to the close/wide degeneracy (Griest \& Safizadeh 1998; Bozza 2000; Dominik 2009). However, close models are slightly favored. The mass ratio of this event is not well constrained. This is due to a lack of observations during the anomaly, especially during the central caustic entrance and exit.

We tried to model second-order effects, such as annual parallax and the orbital motion of the lens (Gould \& Loeb 1992; Dominik 1998; Albrow et al. 2000; Gould 2004; Bachelet et al. 2012b). Due to the relatively short timescale of the event and the relatively low coverage of the anomaly features, these second-order effects were not constrained well enough to be considered a solid detection. 

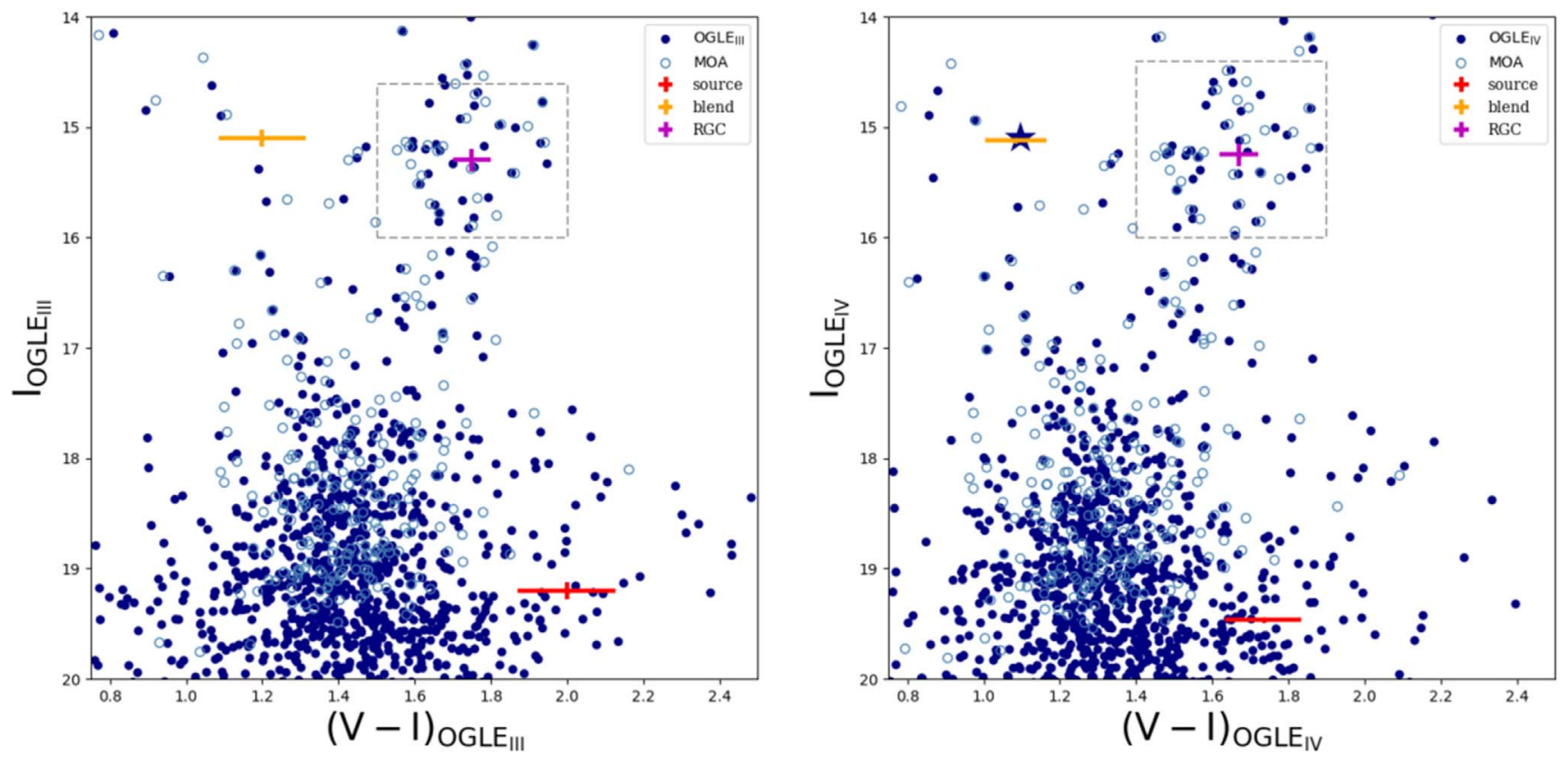

Figure 2. Optical color-magnitude diagrams of stars within 2' of the line of sight of this event. OGLE and the transformed MOA are in blue (filled and empty respectively), the source is in red, the blend is in orange, and the position of the RGC is in magenta. The star symbol represents the star presented in the Table 4. The

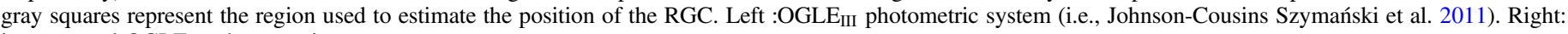
instrumental OGLE $\mathrm{IV}_{\mathrm{V}}$ photometric system.

Table 4

Astrometry of the Target in MOA, OGLE-III, OGLE-IV, VVV, and Gaia Catalogs; the OGLE-III Catalog of the Field is from Szymański et al. (2011)

\begin{tabular}{|c|c|c|c|c|c|c|c|}
\hline Catalog & Source ID & Epoch & R.A.(J2000) & Decl.(J2000) & $\begin{array}{l}\text { Parallax } \\
\text { (mas) }\end{array}$ & $\begin{array}{c}\mu_{\alpha} \\
\left(\operatorname{mas~yr}^{-1}\right)\end{array}$ & $\begin{array}{c}\mu_{\delta} \\
\left(\operatorname{mas~yr}^{-1}\right)\end{array}$ \\
\hline Gaia & 4042761215742767360 & $\mathrm{~J} 2015.5$ & $271.68268633(1)$ & $-32.90760309(1)$ & $0.96(7)$ & $9.9(1)$ & $2.0(1)$ \\
\hline MOA & 965 & $\ldots$ & 271.68263(4) & $-32.90764(3)$ & $\cdots$ & $\cdots$ & $\cdots$ \\
\hline OGLE-III & 90793 & J2002.46 & 271.68267(4) & $-32.90761(3)$ & $\cdots$ & $\cdots$ & $\cdots$ \\
\hline OGLE-IV (baseline) & 58780 & J2011.4 & $271.68267(4)$ & $-32.90758(3)$ & $\cdots$ & $\cdots$ & $\cdots$ \\
\hline OGLE-IV (source) & $\cdots$ & J2011.4 & $271.68269(4)$ & $-32.90720(3)$ & $\cdots$ & $\cdots$ & $\cdots$ \\
\hline VVV & 2508 & J2010 & 271.68262 & -32.90763 & $\cdots$ & $\ldots$ & $\cdots$ \\
\hline
\end{tabular}

Note. The OGLE-IV catalog is available online (http://ogle.astrouw.edu.pl/ogle4/ews/2018/ews.html). The VVV Catalog is from Beaulieu et al. (2016). The PPMXL Catalog is from Roeser et al. (2010). The numbers in parentheses are the $1 \sigma$ uncertainties

\section{Properties of the Source}

\subsection{Optical Observations}

Following Bond et al. (2017), we calibrated the $\mathrm{MOA}_{\mathrm{R}}$ and $\mathrm{MOA}_{\mathrm{V}}$ magnitudes to the OGLE the Appendix. The resulting color-magnitude diagram (CMD thereafter) is presented in Figure 2, and we summarize information from the various catalogs used in Table 4 . We found that the color of the RGC centroid is $(V-I)_{\mathrm{RGC}}=1.75 \pm 0.05 \mathrm{mag}$ and its brightness is $I_{\mathrm{RGC}}=15.3 \pm 0.1 \mathrm{mag}$. Knowing the intrinsic color of the RGC $(V-I)_{0, \mathrm{RGC}}=1.06 \mathrm{mag}$ and its intrinsic brightness $I_{0, \mathrm{RGC}}=14.45 \mathrm{mag}$ (Nataf et al. 2013), we estimate the absorption $A_{I}=0.9 \pm 0.1 \mathrm{mag}$ and the extinction $E(V-I)=0.69 \pm 0.05 \mathrm{mag}$ toward the microlensing event. We found a good agreement with an independent determination using the Interstellar Extinction Calculator on the OGLE website, ${ }^{39}$ based on Nataf et al. (2013) and Gonzalez et al. (2012), with $A_{I}=0.79 \pm 0.1 \mathrm{mag}$ and $E(V-I)=0.68 \pm$ 0.05 mag. From the best model and the color transformations in

\footnotetext{
39 http://ogle.astrouw.edu.pl/
}

the Appendix, the source magnitudes are $V_{\mathrm{s}, \mathrm{OGLE}}=21.2 \pm 0.1$ mag and $I_{\mathrm{s}, \mathrm{OGLE}_{\mathrm{II}}}=19.15 \pm 0.09 \mathrm{mag}$ (and a color of $\left.(V-I)_{\mathrm{s}, \mathrm{OGLE}_{\mathrm{III}}}=2.0 \pm 0.1 \mathrm{mag}\right)$. In principle, it is possible to obtain a model-independent color using linear regression between two bands $\lambda_{1}$ and $\lambda_{2}$ because the microlensing magnification is achromatic (Dong et al. 2006; Bond et al. 2017):

$$
f_{\lambda_{1}}=\frac{f_{s, \lambda_{1}}}{f_{s, \lambda_{2}}}\left(f_{\lambda_{2}}-f_{b, \lambda_{2}}\right)+f_{b, \lambda_{1}}
$$

where $f_{s}$ and $f_{b}$ are the source and blending flux, respectively. However, this requires simultaneous observations, which are difficult in practice. Here, we consider $\mathrm{MOA}_{R}$ and $\mathrm{MOA}_{V}$ as simultaneous if the acquisition time was within 15 minutes. We found a model-independent source color of $(V-I)_{\mathrm{s}, \mathrm{OGLE}_{\mathrm{III}}}=$ $2.0 \pm 0.1 \mathrm{mag}$, in agreement with the previous estimation. Finally, we obtained the intrinsic color $(V-I)_{\mathrm{O}, \mathrm{s}, \mathrm{OGLE}_{\mathrm{III}}}=$ $1.4 \pm 0.1 \mathrm{mag}$ and brightness $I_{\mathrm{O}, \mathrm{s}, \mathrm{OGLE}}=18.4 \pm 0.1$ of the source in the OGLE-III system (i.e., in the Johnson-Cousins system). 
Because this event was also observed by OGLE-IV, we conducted a similar analysis using the OGLE-IV CMD. The corresponding CMD is presented in Figure 2. In this CMD, we found that the color of the red giant clump (RGC) centroid is $(V-I)_{\mathrm{RGC}}=1.67 \pm 0.05 \mathrm{mag}$ and its brightness is $I_{\mathrm{RGC}}=$ $15.3 \pm 0.1 \mathrm{mag}$. The best model and the $V$-band transformation in Equation (8) lead to $V_{\mathrm{s}, \mathrm{OGLE}_{\mathrm{IV}}}=21.2 \pm 0.1 \mathrm{mag}$ and $I_{\mathrm{s}, \mathrm{OGLE}_{\mathrm{IV}}}=$ $19.47 \pm 0.01 \mathrm{mag}$ (and a color of $(V-I)_{\mathrm{s}, \mathrm{OGLE}_{\mathrm{IV}}}=1.7 \pm$ $0.1 \mathrm{mag}$ ). Assuming the source suffers the same extinction as the RGC, we measured an offset between the source and the RGC $\Delta\left((V-I)_{\mathrm{s}, \mathrm{OGLE}_{\mathrm{IV}}}, I_{\mathrm{s}, \mathrm{OGLE}_{\mathrm{IV}}}\right)=(0.03 \pm 0.1,4.2 \pm 0.1)$. However, the OGLE-IV system is not perfectly calibrated, and the difference in the colors needs to be multiplied by a factor of 0.93 (for CCD 24 of the OGLE camera mosaic; Udalski et al. 2015). Based on the OGLE-IV CMD, the source color is $(V-I)_{\mathrm{o}, \mathrm{s}, \mathrm{OGLE}_{\mathrm{IV}}}=1.09 \pm 0.1 \mathrm{mag}$ and the brightness is $I_{\mathrm{o}, \mathrm{s}, \mathrm{OGLE}_{\mathrm{IV}}}=18.7 \pm 0.1 \mathrm{mag}$.

While the two studies converge to a similar conclusion, we use for the source properties $(V-I)_{\mathrm{o}, \mathrm{s}, \mathrm{OGLE}_{\mathrm{IV}}}=1.09 \pm 0.1$ mag and $I_{\mathrm{o}, \mathrm{s}, \mathrm{OGLE}_{\mathrm{IV}}}=18.7 \pm 0.1 \mathrm{mag}$, because they rely on a single color transformation and also because the color term in Equation (8) is smaller than the one in Equation (6). From optical observations, the source is probably a $\mathrm{K}$ dwarf (Bessell \& Brett 1988), or, potentially, a K subgiant that lies behind the Galactic Bulge.

Using Kervella \& Fouqué (2008) and the optical color, we can obtain the angular source radius $\theta_{*}$. We obtain $13 \%$ precision on $\theta_{*}=0.8 \pm 0.1 \mu \mathrm{as}$. Finally, we can then estimate the angular Einstein ring radius $\theta_{E}=\theta_{*} / \rho=0.8 \pm 0.2$ mas (using the best model) and $\mu=7.0 \pm 3$ mas $/ y r$. This provides one mass and distance constraint to the lens system, as (Gould 2000)

$$
M_{\mathrm{tot}}=\frac{\theta_{E}^{2}}{\kappa \pi_{\mathrm{rel}}},
$$

with $\pi_{\text {rel }}=\frac{1-x}{D_{s} x}$ au, $x=D_{l} / D_{s}$ (the distance to the lens and the source respectively) and the constant $\kappa=8.144$ mas. $M_{\odot}^{-1}$.

\subsection{Near-infrared}

Thanks to VVV observations, we can perform a similar study using $K$-band data and construct a near-infrared CMD, as shown in Figure 3. Gonzalez et al. (2012) provided extinction maps toward the Galactic Bulge. Using their online tool, ${ }^{40}$ we found $A_{K}=0.10 \pm 0.06 \mathrm{mag}$ and $E(J-K)=0.19 \pm 0.11 \mathrm{mag}$. This agrees relatively well with the 3D maps toward the Galactic Bulge of Schultheis et al. (2014; i.e., $E(J-K)=0.30 \pm 0.06$ mag and $A_{K}=0.16 \pm 0.04$ mag assuming the Nishiyama et al. 2009 extinction law). From the best model, the source brightness is $K_{s, s}=17 \pm 1 \mathrm{mag}$ and the blend brightness is $K_{s, b}=13.61 \pm$ 0.03 mag. The relatively low precision on the source magnitude in $K_{s}$ is again due to the lack of observations during the event highmagnification phase of the event. Unfortunately, the maximum observed magnification was only $A \sim 1.6$, while the secondary maximum observed magnification was $A \sim 1.05$. The color of the source is $\left(I_{\mathrm{OGLE}_{\mathrm{IV}}}-K_{\mathrm{VVV}}\right)=2 \pm 1 \mathrm{mag}$, leading to an extinction-corrected color of $\left(I_{\mathrm{OGLE}_{\mathrm{IV}}}-K_{\mathrm{VVV}}\right)_{o}=2.0-A_{I}+$ $A_{K}=1 \pm 1 \mathrm{mag}$, and a magnitude of $K_{0, \mathrm{vVv}}=17 \pm 1 \mathrm{mag}$. Using Bessell \& Brett (1988), we found that this color is consistent with the optical color and corresponds to a K-type source star.

\footnotetext{
${ }^{40}$ http://mill.astro.puc.cl/BEAM/calculator.php
}

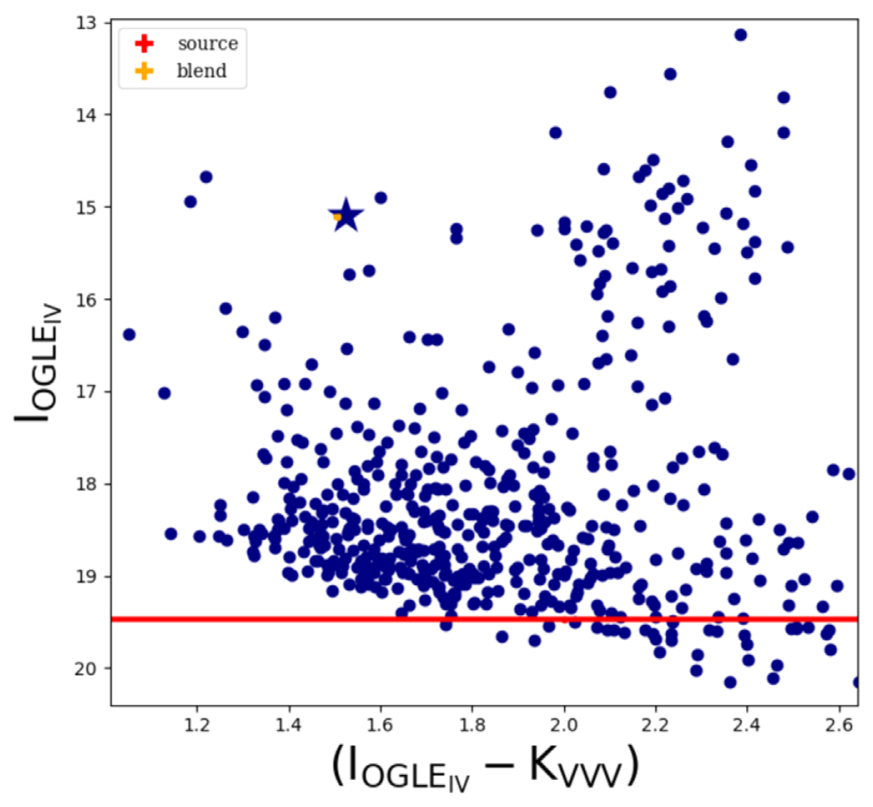

Figure 3. Color-magnitude diagram of stars within $2^{\prime}$ of the line of sight to this event, using $I_{\mathrm{OGLE}}$ and $K_{\mathrm{VVV}}$.

\subsection{Does the Source belong to the Sagittarius Dwarf Galaxy?}

Due to the relatively large galactic latitude of the event (i.e., $\left.b=-5^{\circ} .9060\right)$, the line of sight does not go through much of the Galactic Disk. This raises the possibility that the source is located in the stream of the Sagittarius Dwarf galaxy (Ibata et al. 1994). If this were the case, the source would be located very far away, $D_{s} \sim 25 \mathrm{kpc}$. Cseresnjes \& Alard (2001) predicted that events due to the Sagittarius dwarf galaxy should represent roughly $1 \%$ of the total events detected toward the Galactic Bulge fields each year. They also predicted that these events should mainly occur for main-sequence source stars with $V \geqslant 21 \mathrm{mag}$ and that the median Einstein ring radius crossing time would be 1.3 times larger than the one observed for Milky Way sources. To test this, we constructed a map of the Sagittarius Dwarf galaxy in Figure 4. We followed the method of Majewski et al. (2003) and selected stars with $E(B-V)<0.555, \quad 0.95<\left(J-K_{s}\right)_{o}<1.10$ and $10.5<$ $K_{s, o}<12$ combined them with the extinction maps from Schlegel et al. (1998); with a low resolution of $0.5 \mathrm{deg}){ }^{41}$ However, the line of sight $\left(\ell=-1^{\circ} .17, b=-5^{\circ} .90\right)$ is quite distant from the highest density of the Sagittarius Dwarf galaxy: M54. The Sagittarius dwarf star population has been studied in great detail; see, for example, Marconi et al. (1998), Monaco et al. (2002, 2004), and Giuffrida et al. (2010). Several features can be used to distinguish stars from the Milky Way and the dwarf galaxy. In particular, the CMD of the dwarf galaxy presents several horizontal branches and red-giant branches, signatures of different star populations. The optical CMD of OGLE-2015-BLG-0232 does not show these signatures, indicating that there is no significant contamination from the dwarf galaxy.

Due to the large distance to the center of the Sagittarius Dwarf galaxy $\left(\geqslant 10^{\circ}\right)$ and the absence of particular features in

\footnotetext{
$\overline{41}$ We use the python implementation available at https://github.com/ gregreen/dustmaps.
} 


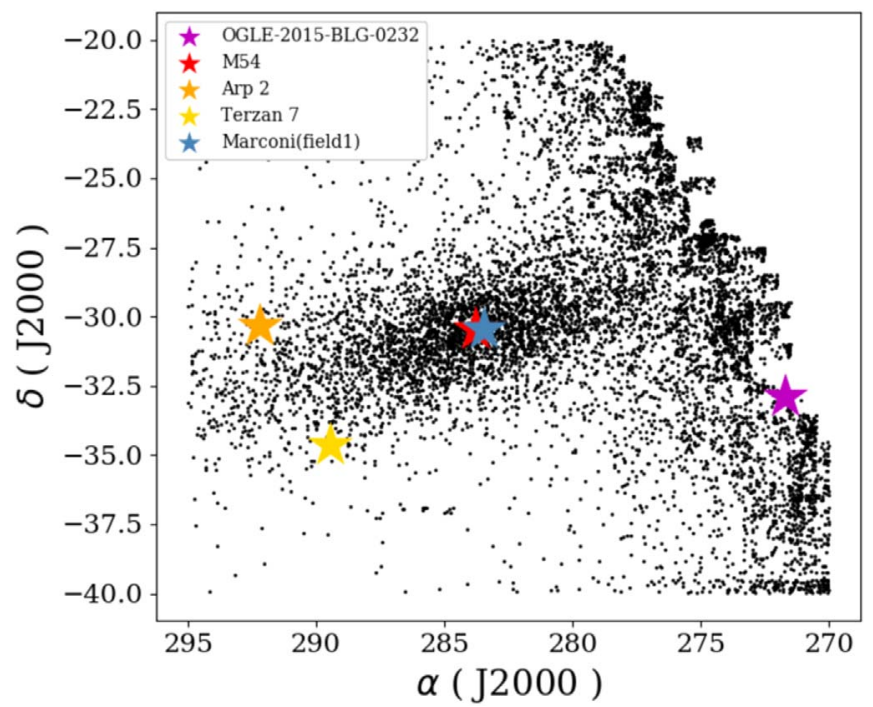

Figure 4. Map of the Sagittarius Dwarf galaxy from the 2MASS catalog (Cutri et al. 2003; Skrutskie et al. 2006).

the CMD, we discount this hypothesis and assume that the source star belongs to the Milky Way.

\section{Information on the Blend}

Results from our modeling indicate that this event was highly blended. It is clear from Figures 2 and 3 that the blend belongs to the foreground stars branch of the CMD, indicating a close blend. In the following, we consider the blend as a single star and neglect the potential contamination from the source because the blend ratio is substantial with $g \sim 50$.

\subsection{Gaia Measurements}

The Gaia mission (Gaia Collaboration et al. 2016, 2018; Luri et al. 2018) recently released a vast catalog of parallax and proper motions measurements for more than a billion of stars. In addition to this goldmine, effective temperatures, radii, and luminosities are also estimated. We summarized the Gaia measurements for OGLE-2015-BLG-0232 in Table 4. Recent studies indicate biases in Gaia parallax measurements of several $\mu$ as (Lindegren et al. 2018; Riess et al. 2018; Zinn et al. 2018). We therefore use the estimation of the blend distance $D_{b}=1023_{-75}^{+86} \mathrm{pc}$ by Bailer-Jones et al. (2018), so the blend is a late-type $\mathrm{G}$ or an early-type $\mathrm{K}$ dwarf. For this target, we also found $T_{\text {eff }}=4707_{-228}^{+269} \mathrm{~K}, R=1.0_{-0.1}^{+0.1} R_{\odot}, \mathcal{L}=0.42_{-0.07}^{+0.07} \mathcal{L}_{\odot}$ and ultimately estimated the mass of this blend as $M \sim \mathcal{L}^{1 / 4} \sim 0.8 M_{\odot}$, which is typical for a $\mathrm{K}$ dwarf. However, Andrae et al. (2018) noted that these parameters are estimated by neglecting the extinction toward the target. While this approximation is reasonable for this target because the blend is relatively close and the extinction along the line of sight is relatively small, these fundamental parameters are probably biased.

The brightnesses of the blend in the Gaia bands are $G=$ $15.918 \pm 0.001 \mathrm{mag}, G_{B P}=16.48 \pm 0.01 \mathrm{mag}$, and $G_{R P}=$ $15.15 \pm 0.01 \mathrm{mag}$. Using the system transformation in the Appendix, we convert these magnitudes to the Johnson-Cousins system to find $V=16.25 \pm 0.05 \mathrm{mag}$ and $I=15.08 \pm 0.05$ mag. Given the blend distance, we assumed half-extinction and found an intrinsic color $(V-I)_{o, b, G}=1.33-0.69 / 2=$ $1.0 \pm 0.1$ and brightness $I_{o, b, G}=15.08-0.79 / 2=14.7 \pm$ $0.1 \mathrm{mag}$, which are typical for a K2 dwarf star (Bessell \& Brett 1988). Using the color-effective temperature relation of Casagrande et al. (2010), the blend effective temperature is $T_{\text {eff }}=4900 \pm 400 \mathrm{~K}$. We estimated the blend physical radius as $R_{b}=0.8 \pm 0.1 R_{\odot}$, and the luminosity as $\mathcal{L}=0.3 \pm 0.1 \mathcal{L}_{\odot}$, and finally derived the blend mass $M_{b} \sim 0.7 M_{\odot}$ (Boyajian et al. 2012). Knowing that the angular radius of the blend is $\theta_{b}=4.8 \pm 0.5 \mu a s$ (Kervella \& Fouqué 2008), one can derive an independent estimate of the blend distance, $D_{b}=800 \pm 200$ pc, in good agreement with the Gaia parallax measurement.

If the blend were the lens and we assume that the source is at $8 \mathrm{kpc}$, with a blend mass of $M_{b} \sim 0.7 M_{\odot}$ at a distance $D_{b} \sim$ $1000 \mathrm{pc}$, the angular Einstein ring would be $\theta_{E, b} \sim 2.2$ mas. This is in strong disagreement with the value of $\theta_{E}=0.8 \pm 0.2$ mas derived in Section 4.1. This is the first clue that the bright blend is likely not the lens.

From Table 4, the proper motion of the bright blend is $\mu_{G}(N, E)=(2.2 \pm 0.1,9.9 \pm 0.1) \operatorname{mas~yr}^{-1}$. The speed of the Sun in the Galactic frame is $V_{\odot}(U, V, W) \sim(11,12,7)+$ $(0,220,0) \mathrm{km} \mathrm{s}^{-1}$ (Fich et al. 1989; Schönrich et al. 2010); the first term is the intrinsic Sun velocity and the second term is the speed of the Galactic disk in the Galactic coordinates system. Assuming the source is at $8 \mathrm{kpc}$, the expected proper motion of the source is about $\boldsymbol{\mu}_{s}(l, b) \sim(-6 \pm 3,0 \pm 3)$ mas yr $^{-1}$; see Kuijken \& Rich (2002) and Kozłowski et al. (2006) for estimation of the uncertainties. The Galactic proper motion transforms to

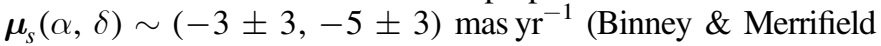
1998; Poleski 2013; Bachelet et al. 2018). Therefore, if the bright blend were the lens, one would expect a relative proper motion of $\boldsymbol{\mu}_{\text {rel }}(N, E)=\mu_{s}-\mu_{G} \sim(-5 \pm 3,-15 \pm 3) \operatorname{mas~yr}^{-1}$. The relative proper motion would be $\mu_{\text {rel }}=16 \pm 4$ mas yr $^{-1}$, in disagreement with the estimation $\mu_{\text {rel }}=7 \pm 3 \mathrm{mas} / \mathrm{yr}$ of the Section 4.1. This is the second clue that the blend is not the lens.

\subsection{Blend Brightness from Models}

Using our best-fit model and the color relationships given in the Appendix, we derived the brightnesses of the blend: $I_{\mathrm{b}, \mathrm{OGLE}}=15.1163 \pm 0.0007 \mathrm{mag}, \mathrm{V}_{\mathrm{b}, \mathrm{OGLE}_{\mathrm{IV}}}=16.23 \pm 0.08$ $\mathrm{mag}$, and $K_{b, \mathrm{VvV}}=13.61 \pm 0.03$ mag. Assuming that the blend suffers half the extinction, we found that the blend brightness is $I_{o, b, \mathrm{OGLE}_{\mathrm{IV}}}=14.7 \pm 0.1 \mathrm{mag}$ and the blend color is $\left(I_{\mathrm{OGLE}_{\mathrm{IV}}}-K_{\mathrm{VVV}}\right)_{o, b}=1.1 \pm 0.1 \mathrm{mag}$, consistent with its being an early K dwarf (Bessell \& Brett 1988). This is in good agreement with the Gaia measurements.

\subsection{Astrometry}

Toward the Galactic Bulge and for stellar masses, microlensing occurs when the alignment between the lens and the source is less than a few mas. We therefore compared the position of centroids between the baseline object and the magnified source from the OGLE-IV images. In pixel coordinates, the magnified source has an offset of $\Delta(N, E)=(78 \pm 45,78 \pm 35)$ mas from the bright blend centroid (the precision of the bright blend centroid is about 0.05 pixel, i.e., $\sigma(N, E)=(13,13)$ mas $)$. The two positions are 
different enough (i.e., $1.5 \sigma$ ) to assume that this is the third clue that the blend is likely not the lens.

\subsection{The Lens as a Blend Companion}

In the following, we explore the possibility that the lens is a companion of the blend. From the astrometry offset derived in Section 5.3, we can derive the separation $\delta$ of the blend with its potential companion and found $\delta=110$ mas, which corresponds to $\mathrm{a}_{\text {proj }} \sim 110 \mathrm{au}$ at $1 \mathrm{kpc}$. If this potential companion is indeed a component of the lens system, then the mass ratio between the binary blend components is $q_{b}=\left(\theta_{E} / \theta_{E, b}\right)^{2}=$ $(0.8 / 2.2)^{2} \sim 0.13$, leading to a potential companion mass of $M_{b, 2}=0.13 \times 0.7 \sim 0.1 M_{\odot}$. Therefore, such a companion is not bright enough to have been significantly detected. Because the normalized separation between the putative companion and the bright blend is important $s_{b}=110 / 2.2 \sim 50$, the hypothetic companion blend could have acted as a binary lens and left no signature of a triple lens, as observed. However, this hypothetic companion would have a similar proper motion, as the bright blend and the analysis on the relative proper motions in Section 5.1 also apply here. Therefore, the lens as a blend companion hypothesis is unlikely.

\section{Discussion and Potential New Clues}

All available information seems to concur that the blend light is mainly due to a close $\mathrm{K}$ dwarf. Both astrometry and the constraint from finite-source effects reject the hypothesis that the bright blend is the lens. The light of the lens is not significantly detected and there are no constraints from the microlensing parallax: the distance and exact nature of the lens remain uncertain for now. However, considering a large mass range for the lens primary $M_{l, 1} \in[0.1,2.0] M_{\odot}$ (corresponding to $D_{l} \leqslant 5.5 \mathrm{kpc}$ according to Equation (4) and $\theta_{E}=0.8 \pm 0.2$ mas), the companion mass range is $M_{l, 2} \in[6,130] M_{\mathrm{Jup}}$. The lens companion is therefore a massive planet, a brown dwarf, or a low-mass $\mathrm{M}$ dwarf if $D_{l} \leqslant 5.5 \mathrm{kpc}$. It the lens is more distant, the primary is probably a stellar remnant, otherwise the lens light would have been detected. This indicates the need for supplementary observations to reveal the nature of the lens OGLE-2015-BLG-0232.

High-resolution imaging is an important tool for microlensing. Several planets have been confirmed using space-based or ground-based facilities and had their measured properties refined (see, for example, Batista et al. 2015; Bennett et al. 2015; and Beaulieu et al. 2018). High-resolution imaging is useful for two reasons. First, it is possible to estimate the source-lens proper motion $\mu$ from high-resolution images obtained several years after the microlensing event, when the source and the lens are well separated (Batista et al. 2015). High-resolution imaging can also provide measurements of a source and sometimes lens fluxes, therefore tightly constraining the mass-distance relation of the lens (Batista et al. 2015; Bennett et al. 2015; Ranc et al. 2015; Beaulieu et al. 2018).

In the case of OGLE-2015-BLG-0232, high-resolution imaging will contribute to confirming/rejecting scenarios and possibly estimating the mass of the lens. The first step will be to challenge the assumption that the blend is a single star. This can be done immediately. Moreover, one can predict a more precise source position based on Gaia astrometry and the measured offset from the OGLE-IV photometry. The predicted

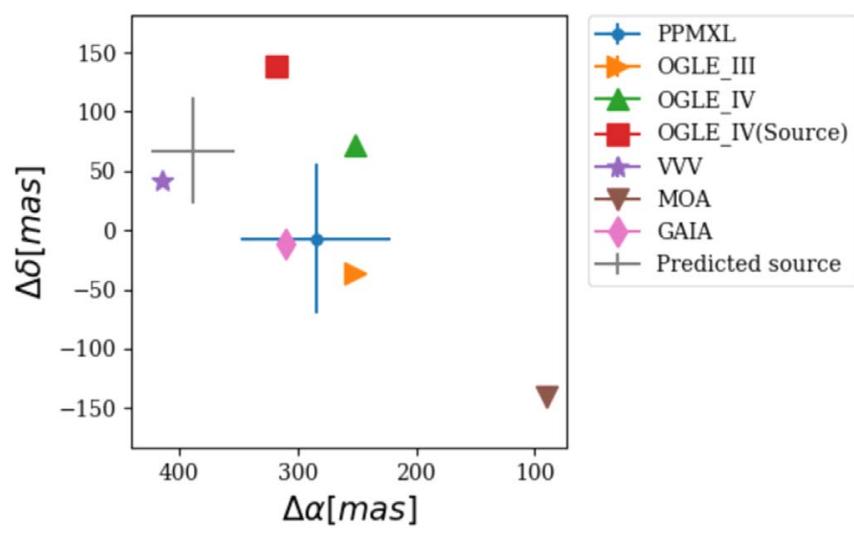

Figure 5. Location of the target from the catalogs listed in the Table 4, centered at $\left(\right.$ R.A. $=271.6826^{\circ}$, Decl. $\left.=-32.9076^{\circ}\right)(\mathrm{J} 2000)$. North is up and east is left. Some uncertainties have been hidden for clarity. The source position measured from OGLE-IV is located at $\Delta(E, N)=(78,78)$ mas (red square) from the OGLE-IV position. The gray cross indicates the prediction of the source, applying the offset to the Gaia position and assuming $\sigma(N, E)=$ $(45,35)$ mas uncertainties.

position of the source is shown in Figure 5, assuming 26 mas precision on the OGLE-IV measurement (i.e., 0.1 pixel). The comparison of the flux at this position in high-resolution images with the measured source fluxes from models, could place constraints on the nature of the lens.

A second step will be to wait several years for the bright blend leaves the line of sight to obtain more information on the source/lens system. Because $\mu_{b}=11 \pm 0.2 \mathrm{mas} \mathrm{yr}^{-1}$, the blend is separating faster than the lens/source system

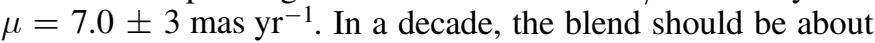
11 pixels away from the line of sight, while the source and the lens separation should be about 7 pixels (for a typical highresolution pixel scale of 10 mas pix $^{-1}$ ).

Low-resolution spectroscopy could also confirm the spectral type of the bright blend. Similarly, the study of emission/ absorption lines with high-resolution spectroscopy would allow a precise understanding of the blend. Finally, one could combine spectroscopic and photometric information to explore various scenarios in a Bayesian analysis (Santerne et al. 2016).

\section{Conclusion}

We presented an analysis of the binary microlensing event OGLE-2015-BLG-0232. Because the event occurred during full moon, the observations do not constrain the deviations from the single-lens model. However, results from the modeling favor a close brown dwarf companion (i.e., $s \sim 0.55$ and $q \sim 0.06$ ). The source is estimated to be red and faint, probably a $\mathrm{K}$ dwarf in the Galactic Bulge. We also tested, and ultimately rejected, the hypothesis that the source belongs to the Sagittarius Dwarf Galaxy. Since the microlensing parallax is not measured, we obtain only one (weak) constraint, from finite-source effects, on the mass and distance of the lens. Based on the recent Gaia DR2 release and OGLEIV astrometry, we were able to infer that the bright blend is a $\mathrm{K}$ dwarf at $1 \mathrm{kpc}$ and is most likely not the lens. We finally discuss the potential of additional observations to confirm the nature of the blend and ultimately to derive the exact nature of the lens. 
The authors thank the anonymous referee for the constructive comments. This research has made use of NASA's Astrophysics Data System. Work by E.B. and R.A.S. is support by the NASA grant NNX15AC97G. Work by C. Han was supported by the grant (2017R1A4A1015178) of National Research Foundation of Korea. This work makes use of observations from the LCOGT network. The OGLE project has received funding from the National Science Centre, Poland, grant MAESTRO 2014/14/A/ST9/ 00121 to A.U. The MOA project is supported by JSPS KAKENHI grants No. JSPS24253004, JSPS26247023, JSPS23340064, JSPS15H00781, JP16H06287, and JP17H02871. The work by C.R. was supported by an appointment to the NASA Postdoctoral Program at the Goddard Space Flight Center, administered by USRA through a contract with NASA. This work has made use of data from the European Space Agency (ESA) mission Gaia (https://www.cosmos.esa.int/Gaia), processed by the Gaia Data Processing and Analysis Consortium (DPAC, https://www. cosmos.esa.int/web/Gaia/dpac/consortium). Funding for the DPAC has been provided by national institutions, in particular the institutions participating in the Gaia Multilateral Agreement. This publication makes use of data products from the Two Micron All Sky Survey, which is a joint project of the University of Massachusetts and the Infrared Processing and Analysis Center/ California Institute of Technology, funded by the National Aeronautics and Space Administration and the National Science Foundation. This research made use of Astropy, a communitydeveloped core Python package for Astronomy (Astropy Collaboration, 2013). This research has made use of the SIMBAD database, operated at CDS, Strasbourg, France. The DENIS project has been partly funded by the SCIENCE and HCM plans of the European Commission under grants CT920791 and CT940627. It is supported by INSU, MEN, and CNRS in France, by the State of Baden-Württemberg in Germany, by DGICYT in Spain, by CNR in Italy, by FFwFBWF in Austria, by FAPESP in Brazil, by OTKA grants F-4239 and F-013990 in Hungary, and by the ESO C\&EE grant A-04-046. Jean Claude Renault from IAP was the Project manager. Observations were carried out thanks to the contribution of numerous students and young scientists from all involved institutes, under the supervision of P. Fouqué, a survey astronomer resident in Chile. D.P.B., A.B., and C.R. were supported by NASA through grant NASA-80NSSC18K0274.

\section{Appendix Color Transformations}

In this work, we used several color transformations that we summarize here. First, we calibrated the MOA instrumental magnitudes to the OGLE-III catalog (Udalski 2003; Bond et al. 2017) using the relationships

$$
\begin{aligned}
I_{\mathrm{OGLE}_{\mathrm{III}}}= & R_{\mathrm{MOA}}+(27.935 \pm 0.003) \\
& +(-0.244 \pm 0.003)\left(V_{\mathrm{MOA}}-R_{\mathrm{MOA}}\right) \pm 0.08, \\
V_{\mathrm{OGLE}_{\mathrm{III}}=} & V_{\mathrm{MOA}}+(28.556 \pm 0.002) \\
& +(-0.164 \pm 0.002)\left(V_{\mathrm{MOA}}-R_{\mathrm{MOA}}\right) \pm 0.08 .
\end{aligned}
$$

We also calibrated the MOA instrumental magnitudes to the OGLE-IV system using

$$
\begin{aligned}
I_{\mathrm{OGLE}_{\mathrm{IV}}}= & R_{\mathrm{MOA}}+(27.990 \pm 0.003) \\
& +(-0.247 \pm 0.009)\left(V_{\mathrm{MOA}}-R_{\mathrm{MOA}}\right) \pm 0.08,
\end{aligned}
$$

$$
\begin{aligned}
V_{\mathrm{OGLE}_{\mathrm{IV}}}= & V_{\mathrm{MOA}}+(28.425 \pm 0.005) \\
& +(-0.062 \pm 0.006)\left(V_{\mathrm{MOA}}-R_{\mathrm{MOA}}\right) \pm 0.08
\end{aligned}
$$

We also used the transformation of the 2MASS colors into the the VVV system (Soto et al. 2013):

$$
\begin{gathered}
J_{\mathrm{VVV}}=J_{2 \mathrm{MASS}}-0.077\left(J_{2 \mathrm{MASS}}-H_{2 \mathrm{MASS}}\right), \\
H_{\mathrm{VVV}}=H_{2 \mathrm{MASS}}+0.032\left(J_{2 \mathrm{MASS}}-H_{2 \mathrm{MASS}}\right), \\
K_{\mathrm{VVV}}=K_{2 \mathrm{MASS}}+0.010\left(J_{2 \mathrm{MASS}}-K_{2 \mathrm{MASS}}\right) .
\end{gathered}
$$

Transformations into the Bessell \& Brett photometric system (Bessell \& Brett 1988) are the revised version ${ }^{42}$ of Carpenter (2001):

$$
\begin{aligned}
&\left(K_{s}\right)_{2 \mathrm{MASS}}= K_{\mathrm{BB}}+(-0.039 \pm 0.007) \\
&+(0.001 \pm 0.005)(J-K)_{\mathrm{BB}} \\
&\left(J-K_{s}\right)_{2 \mathrm{MASS}}=(-0.018 \pm 0.007) \\
&+(0.001 \pm 0.005)(J)_{\mathrm{BB}} .
\end{aligned}
$$

Finally, the transformation of the Gaia DR2 to the JohnsonCousins system is available online ${ }^{43}$ :

$$
\begin{aligned}
G-V_{\mathrm{JC}}= & -0.01760-0.006860\left(G_{\mathrm{BP}}-G_{\mathrm{RP}}\right) \\
& -0.1732\left(G_{\mathrm{BP}}-G_{\mathrm{RP}}\right)^{2} \pm 0.045858 \\
G-I_{\mathrm{JC}}= & 0.02085+0.7419\left(G_{\mathrm{BP}}-G_{\mathrm{RP}}\right) \\
& -0.096311\left(G_{\mathrm{BP}}-G_{\mathrm{RP}}\right)^{2} \pm 0.04956 .
\end{aligned}
$$

\section{ORCID iDs}

E. Bachelet (1D https://orcid.org/0000-0002-6578-5078

V. Bozza (iD https://orcid.org/0000-0003-4590-0136 J.-P. Beaulieu (i) https://orcid.org/0000-0003-0014-3354

R. A. Street (iD https://orcid.org/0000-0001-6279-0552

K. Horne (iD https://orcid.org/0000-0003-1728-0304

M. Hundertmark (i) https://orcid.org/0000-0003-0961-5231

C. Ranc (i) https://orcid.org/0000-0003-2388-4534

C. Snodgrass (iD https://orcid.org/0000-0001-9328-2905

I. A. Steele (iD https://orcid.org/0000-0001-8397-5759

Y. Tsapras (ib https://orcid.org/0000-0001-8411-351X

J. Wambsganss (iD https://orcid.org/0000-0002-8365-7619

P. Mróz (10 https://orcid.org/0000-0001-7016-1692

J. Skowron (1) https://orcid.org/0000-0002-2335-1730

P. Pietrukowicz (1D) https://orcid.org/0000-0002-2339-5899

K. Ulaczyk (ib https://orcid.org/0000-0001-6364-408X

A. Fukui (iD https://orcid.org/0000-0002-4909-5763

Y. Itow (i) https://orcid.org/0000-0002-8198-1968

N. Koshimoto (1) https://orcid.org/0000-0003-2302-9562

S. Miyazaki (i) https://orcid.org/0000-0001-9818-1513

N. J. Rattenbury (i) https://orcid.org/0000-0001-5069-319X

D. Suzuki iD https://orcid.org/0000-0002-5843-9433

\section{References}

Albrow, M. D., Beaulieu, J.-P., Caldwell, J. A. R., et al. 2000, ApJ, 535, 176 Albrow, M. D., Horne, K., Bramich, D. M., et al. 2009, MNRAS, 397, 2099 Andrae, R., Fouesneau, M., Creevey, O., et al. 2018, A\&A, 616, A8 Andrae, R., Schulze-Hartung, T., \& Melchior, P. 2010, arXiv:1012.3754 Bachelet, E., Beaulieu, J.-P., Boisse, I., Santerne, A., \& Street, R. A. 2018, ApJ, 865, 162

Bachelet, E., Bramich, D. M., Han, C., et al. 2015, ApJ, 812, 136

\footnotetext{
42 https://www.ipac.caltech.edu/2mass/releases/allsky/doc/sec6_4b.html

43 https://gea.esac.esa.int/archive/documentation/GDR2/
} 
Bachelet, E., Fouqué, P., Han, C., et al. 2012a, A\&A, 547, A55

Bachelet, E., Norbury, M., Bozza, V., \& Street, R. 2017, AJ, 154, 203

Bachelet, E., Shin, I.-G., Han, C., et al. 2012b, ApJ, 754, 73

Bailer-Jones, C. A. L., Rybizki, J., Fouesneau, M., Mantelet, G., \& Andrae, R. 2018, AJ, 156, 58

Batista, V., Beaulieu, J.-P., Bennett, D. P., et al. 2015, ApJ, 808, 170

Beaulieu, J.-P., Batista, V., Bennett, D. P., et al. 2018, AJ, 155, 78

Beaulieu, J.-P., Bennett, D. P., Batista, V., et al. 2016, ApJ, 824, 83

Bennett, D. P., Bhattacharya, A., Anderson, J., et al. 2015, ApJ, 808, 169

Bessell, M. S., \& Brett, J. M. 1988, PASP, 100, 1134

Binney, J., \& Merrifield, M. 1998, Galactic Astronomy (Princeton, NJ: Princeton Univ. Press)

Bond, I. A., Abe, F., Dodd, R. J., et al. 2001, MNRAS, 327, 868

Bond, I. A., Bennett, D. P., Sumi, T., et al. 2017, MNRAS, 469, 2434

Bonfils, X., Delfosse, X., Udry, S., et al. 2013, A\&A, 549, A109

Boyajian, T. S., von Braun, K., van Belle, G., et al. 2012, ApJ, 757, 112

Bozza, V. 2000, A\&A, 359, 1

Bozza, V. 2010, MNRAS, 408, 2188

Bozza, V., Dominik, M., Rattenbury, N. J., et al. 2012, MNRAS, 424, 902

Bramich, D. M. 2008, MNRAS, 386, L77

Bramich, D. M., Horne, K., Albrow, M. D., et al. 2013, MNRAS, 428, 2275

Bryan, M. L., Benneke, B., Knutson, H. A., Batygin, K., \& Bowler, B. P. 2018,

NatAs, 2, 138

Buchhave, L. A., Latham, D. W., Johansen, A., et al. 2012, Natur, 486, 375

Burrows, A., Hubbard, W. B., Lunine, J. I., \& Liebert, J. 2001, RvMP, 73, 719

Burrows, A., \& Liebert, J. 1993, RvMP, 65, 301

Carpenter, J. M. 2001, AJ, 121, 2851

Casagrande, L., Ramírez, I., Meléndez, J., Bessell, M., \& Asplund, M. 2010, A\&A, 512, A54

Cassan, A. 2008, A\&A, 491, 587

Cassan, A., Kubas, D., Beaulieu, J.-P., et al. 2012, Natur, 481, 167

Chung, S.-J., Zhu, W., Udalski, A., et al. 2017, ApJ, 838, 154

Clanton, C., \& Gaudi, B. S. 2016, ApJ, 819, 125

Cseresnjes, P., \& Alard, C. 2001, A\&A, 369, 778

Cutri, R. M., Skrutskie, M. F., van Dyk, S., et al. 2003, yCat, 2246

Dominik, M. 1998, A\&A, 329, 361

Dominik, M. 1999, A\&A, 349, 108

Dominik, M. 2009, MNRAS, 393, 816

Dominik, M., Bachelet, E., Bozza, V., et al. 2018, arXiv:1808.03149

Dominik, M., Rattenbury, N. J., Allan, A., et al. 2007, MNRAS, 380, 792

Dong, S., DePoy, D. L., Gaudi, B. S., et al. 2006, ApJ, 642, 842

Erdl, H., \& Schneider, P. 1993, A\&A, 268, 453

Fich, M., Blitz, L., \& Stark, A. A. 1989, ApJ, 342, 272

Foreman-Mackey, D., Hogg, D. W., Lang, D., \& Goodman, J. 2013, PASP, 125,306

Fressin, F., Torres, G., Charbonneau, D., et al. 2013, ApJ, 766, 81

Gaia Collaboration, Brown, A. G. A., Vallenari, A., et al. 2018, arXiv:1804. 09365

Giuffrida, G., Sbordone, L., Zaggia, S., et al. 2010, A\&A, 513, A62

Gonzalez, O. A., Rejkuba, M., Zoccali, M., et al. 2012, A\&A, 543, A13

Gould, A. 2000, ApJ, 542, 785

Gould, A. 2004, ApJ, 606, 319

Gould, A., \& Loeb, A. 1992, ApJ, 396, 104

Griest, K., \& Safizadeh, N. 1998, ApJ, 500, 37

Han, C. 2009, ApJL, 691, L9

Han, C., Jung, Y. K., Udalski, A., et al. 2016, ApJ, 822, 75
Hundertmark, M., Street, R. A., Tsapras, Y., et al. 2018, A\&A, 609, 55 Ibata, R. A., Gilmore, G., \& Irwin, M. J. 1994, Natur, 370, 194

Kervella, P., \& Fouqué, P. 2008, A\&A, 491, 855

Kozłowski, S., Woźniak, P. R., Mao, S., et al. 2006, MNRAS, 370, 435

Kuijken, K., \& Rich, R. M. 2002, AJ, 124, 2054

Latham, D. W., Stefanik, R. P., Torres, G., et al. 2002, AJ, 124, 1144

Liebig, C., D’Ago, G., Bozza, V., \& Dominik, M. 2015, MNRAS, 450, 1565

Lindegren, L., Hernández, J., Bombrun, A., et al. 2018, A\&A, 616, A2

Luri, X., Brown, A. G. A., Sarro, L. M., et al. 2018, A\&A, 616, 9

Majewski, S. R., Skrutskie, M. F., Weinberg, M. D., \& Ostheimer, J. C. 2003, ApJ, 599, 1082

Mao, S., \& Di Stefano, R. 1995, ApJ, 440, 22

Marconi, G., Buonanno, R., Castellani, M., et al. 1998, A\&A, 330, 453

Minniti, D., Lucas, P. W., Emerson, J. P., et al. 2010, NewA, 15, 433

Miyake, N., Udalski, A., Sumi, T., et al. 2012, ApJ, 752, 82

Monaco, L., Bellazzini, M., Ferraro, F. R., \& Pancino, E. 2004, MNRAS, 353,874

Monaco, L., Ferraro, F. R., Bellazzini, M., \& Pancino, E. 2002, ApJL, 578, L47

Mordasini, C., Alibert, Y., Benz, W., Klahr, H., \& Henning, T. 2012, A\&A, 541, A97

Mortier, A., Santos, N. C., Sozzetti, A., et al. 2012, A\&A, 543, A45

Mróz, P., Han, C., Udalski, A., et al. 2017, AJ, 153, 143

Nataf, D. M., Gould, A., Fouqué, P., et al. 2013, ApJ, 769, 88

Nishiyama, S., Tamura, M., Hatano, H., et al. 2009, ApJ, 696, 1407

Penny, M. T. 2014, ApJ, 790, 142

Poleski, R. 2013, arXiv:1306.2945

Poleski, R., Udalski, A., Bond, I. A., et al. 2017, A\&A, 604, A103

Gaia Collaboration, Prusti, T., de Bruijne, J. H. J., et al. 2016, A\&A, 595, A1

Ranc, C., Cassan, A., Albrow, M. D., et al. 2015, A\&A, 580, A125

Riess, A. G., Casertano, S., Yuan, W., et al. 2018, ApJ, 861, 126

Roeser, S., Demleitner, M., \& Schilbach, E. 2010, AJ, 139, 2440

Santerne, A., Beaulieu, J.-P., Rojas Ayala, B., et al. 2016, A\&A, 595, L11

Schlaufman, K. C. 2018, ApJ, 853, 37

Schlegel, D. J., Finkbeiner, D. P., \& Davis, M. 1998, ApJ, 500, 525

Schneider, J., Dedieu, C., Le Sidaner, P., Savalle, R., \& Zolotukhin, I. 2011, A\&A, 532, A79

Schönrich, R., Binney, J., \& Dehnen, W. 2010, MNRAS, 403, 1829

Schultheis, M., Chen, B. Q., Jiang, B. W., et al. 2014, A\&A, 566, A120

Shvartzvald, Y., Yee, J. C., Skowron, J., et al. 2018, arXiv:1805.08778

Skowron, J., Udalski, A., Kozłowski, S., et al. 2016, AcA, 66, 1

Skrutskie, M. F., Cutri, R. M., Stiening, R., et al. 2006, AJ, 131, 1163

Soto, M., Barbá, R., Gunthardt, G., et al. 2013, A\&A, 552, A101

Spiegel, D. S., Burrows, A., \& Milsom, J. A. 2011, ApJ, 727, 57

Storn, R., \& Price, K. 1997, J. Global Optimization, 11, 341

Suzuki, D., Bennett, D. P., Sumi, T., et al. 2016, ApJ, 833, 145

Szymański, M. K., Udalski, A., Soszyński, I., et al. 2011, AcA, 61, 83

Tsapras, Y., Street, R., Horne, K., et al. 2009, AN, 330, 4

Udalski, A. 2003, AcA, 53, 291

Udalski, A., Szymański, M. K., \& Szymański, G. 2015, AcA, 65, 1

Wright, J. T., Fakhouri, O., Marcy, G. W., et al. 2011, PASP, 123, 412

Yee, J. C., Hung, L.-W., Bond, I. A., et al. 2013, ApJ, 769, 77

Zapolsky, H. S., \& Salpeter, E. E. 1969, ApJ, 158, 809

Zhu, W., Calchi Novati, S., Gould, A., et al. 2016, ApJ, 825, 60

Zinn, J. C., Pinsonneault, M. H., Huber, D., \& Stello, D. 2018, arXiv:1805. 02650 